\title{
Arteriovenous malformation and thyroid metastasis from underlying renal cell carcinoma, an unusual presentation of malignancy: A case report
}

\author{
H. J. ALBANDAR ${ }^{1}$, E. S. ROBERTO ${ }^{1}$, J. R. H. SEE ${ }^{2}$ and J. H. SABIERS ${ }^{2}$ \\ ${ }^{1}$ Department of Internal Medicine; ${ }^{2}$ Division of Hematology Oncology, \\ Wright State University Boonshoft School of Medicine, Dayton, OH 45409, USA
}

Received May 25, 2016; Accepted November 17, 2016

DOI: $10.3892 / \mathrm{ol} .2017 .5822$

\begin{abstract}
Renal Clear Cell Carcinoma (RCC) comprises over $80 \%$ of renal malignancies in adults. Thyroid gland metastasis is rare in RCC. Few studies have described cases of RCC mistaken for benign arteriovenous malformation (AVM). To the best of our knowledge, an AVM arising from underlying RCC metastasis to the brain has not yet been reported. The current study presents a case of RCC metastasis to the thyroid gland, with an AVM identified to be a result of metastatic involvement in the brain. A 45-year-old African-American female presented with left-sided weakness, slurred speech, facial droop and seizure. The patient's medical history was notable for a diagnosis of RCC, 2010 American Joint Committee on Cancer Tumor-Node-Metastasis Stage 1B (T1B, N0, M0) grade III status post-right partial nephrectomy. Computed tomography (CT) imaging revealed a soft-tissue mass, suspected to be metastasis, in the left lobe of the thyroid, in addition to a $1.9 \mathrm{~cm}$ right intracranial mass in the parietal lobe. Positron emission tomography/computed tomography revealed a hypermetabolic area in the thyroid. Fine needle aspiration of the thyroid, and subsequent histopathological analysis, suggested a diagnosis of RCC metastasis. Subsequent immunohistochemical analysis of the thyroid tumor confirmed RCC metastasis. The patient also underwent a right partial craniotomy with resection of the intra-axial mass. Initial pathology was suggestive of an AVM. After several months, the patient was readmitted with headache, nausea and vomiting. Repeat imaging revealed recurrence of a $3.9 \mathrm{~cm}$ mass that was negative for AVM on biopsy; however, the immunostaining markers were positive for RCC. Recent literature suggests a link between AVMs and RCC as each
\end{abstract}

Correspondence to: Dr H. J. Albandar, Department of Internal Medicine, Wright State University Boonshoft School of Medicine, 1 Wyoming Street, Dayton, OH 45409, USA

E-mail: heidar.albandar@wright.edu

Key words: renal cell cancer, arteriovenous malformation, thyroid metastasis, presentation, unusual exhibit highly vascular characteristics. RCC is a particularly vascular tumor that has been demonstrated to lead to the abnormal expression of various angiogenesis-promoting growth factors, including vascular endothelial growth factor. These angiogenic factors are vital to the pathophysiological pathway involved in the tumorigenesis and progression of RCC, and may explain the development of AVMs within these neoplasms, as demonstrated in the case presented in the current study.

\section{Introduction}

Renal Clear Cell Carcinoma (RCC) comprises $>80 \%$ of renal malignancies in adults (1). Common sites of RCC metastasis include the lung, bone, liver and adrenal glands (2). The thyroid gland, however, represents a less common metastatic target site for renal malignancies in general, and RCC in particular (2). The incidence of metastasis to the thyroid was revealed to occur in $1.25-24 \%$ of autopsy cases (2). According to the US National Cancer Institute (Rockville, MD, USA), the five-year survival rate for patients diagnosed with renal cancer is $\sim 73.2 \%$, and this number decreases to $\sim 11 \%$ with proven metastasis (3).

There have been several reports in the literature of acquired arteriovenous malformations (AVM) being mistaken for RCC (4). Conversely, RCCs have also been mistaken for AVMs (5). Radiologically, an AVM may take numerous forms, with masses ranging from cystic to solid necrotic, from homogeneous to heterogeneous and from small to large (6). To the best of our knowledge, an AVM associated with RCC metastasis to the brain has never been reported in the literature. The typical clinical presentation of an AVM includes the cardinal features of hemorrhage, seizures, progressive neurological deficits or headache (7). However, recent animal models have postulated that genetic manipulation and angiogenic stimulation, including malignancy, are required for AVM development and are independent of congenital etiologies (8). The current study presents a case of RCC metastasis to the thyroid gland, with an AVM associated with metastatic involvement in the brain. This case is unique as the metastasis to the thyroid was only detected due to the clinical manifestations of a cerebral AVM. 
Case investigations, research and writing were conducted at the Miami Valley Hospital in Dayton Ohio, a teaching institution for Wright State University Boonshoft School of Medicine. The case presented is unique in that it informs the academic community of a novel presentation of renal cell carcinoma that has metastasized to the thyroid and brain. Furthermore, it demonstrates how a presentation of what appears to be an AVM is anything but. Lastly, the metastasis of RCC to the thyroid is a rare event that was further unusual in this case in that it was detected only by diagnosis of the 'AVM'.

\section{Case report}

A 45-year-old African-American female presented to the Miami Valley Hospital Emergency Department (Dayton, OH, USA) in November 2015 with left-sided weakness, slurred speech, facial droop and a grand-mal seizure. The patient's medical history was notable for a diagnosis of RCC, 2010 American Joint Committee on Cancer Tumor-Node-Metastasis Stage 1B (T1B, N0, M0) grade III status post-right robotic-assisted partial nephrectomy 18 months prior. There was no personal or family history of seizure disorders or neurologic diseases. A review of subjective symptoms was negative for vision changes, fevers, chills, weight changes, unilateral weakness, or decreased sensation. Physical examination revealed an unresponsive patient with spontaneous movement of the right side, but flaccid paralysis of the left upper and lower extremities. No thyromegaly, thyroid nodules, cervical or submandibular lymph nodes were palpated. Laboratory tests revealed that blood, liver and kidney function were normal. Magnetic resonance imaging (MRI) of the cervical, thoracic and lumbar spine revealed signs of a metastatic lesion. The patient had numerous episodes of focal status epileptics during hospitalization. After several days, the patient underwent a right partial parietal craniotomy with resection of the intra-axial mass; subsequent pathology was inconclusive for a malignancy and consistent with an AVM.

Due to the thyroid mass observed on the MRI, a positron emission tomography (PET)/computed tomography (CT) scan was performed, which revealed a hypermetabolic area in the left lobe of the thyroid extending into the left aspect of the isthmus. A corresponding ultrasound of the thyroid identified a homogeneous hypervascular lesion throughout the left lobe that extended into the isthmus and had poorly-defined borders. Fine needle aspiration of the thyroid and subsequent histopathological analysis revealed the thyroid tumor to be comprised of clear cell carcinoma cells. Immunohistochemical analysis (Figs. 1-4) of 10\% formalin-fixed paraffin-embedded aspirate tissue sections (4- $\mu \mathrm{m}$ thick) of the thyroid tumor revealed the following: Polyclonal cytokeratins 1/3 (AE1/3; 1:450 dilution; catalog no. M3515; Agilent Technologies, Inc., Santa Clara, CA, USA), positive; vimentin, positive; paired box gene 8 (PAX8; 1:400 dilution; catalog no. 363M-16; Cell Marque, Rocklin, CA, USA), positive; renal cell carcinoma (PN-15; 1:300 dilution; catalog no. 329M-97; Cell Marque), positive; cluster of differentiation (CD) 10 (1:10 dilution; catalog no. AC-0169; Epitomics, Burlingame, CA, USA), positive; cytokeratin 20 (CK20), negative; cytokeratin 7 (CK7), negative; thyroglobulin, negative; and thyroid transcription factor-1, negative.

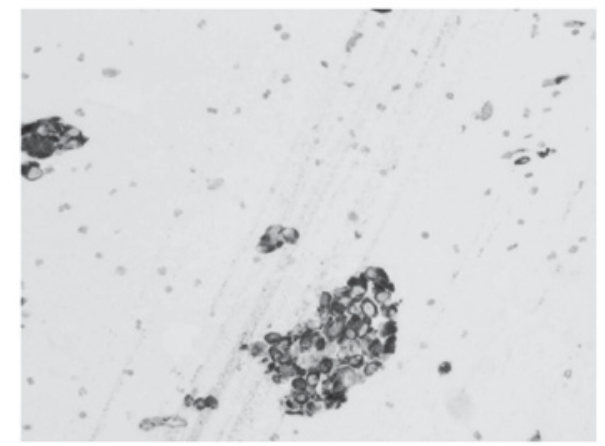

Figure 1. Pathological examination of the thyroid gland resected during surgery. Polyclonal cytokeratins $1 / 3+$ staining (magnification, x200).

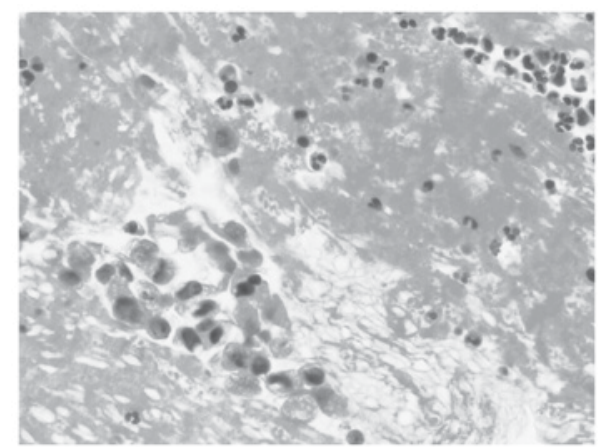

Figure 2. Pathological examination of the thyroid gland resected during surgery. Cluster of differentiation 10 immunohistochemical staining (magnification, $\mathrm{x} 200$ ).

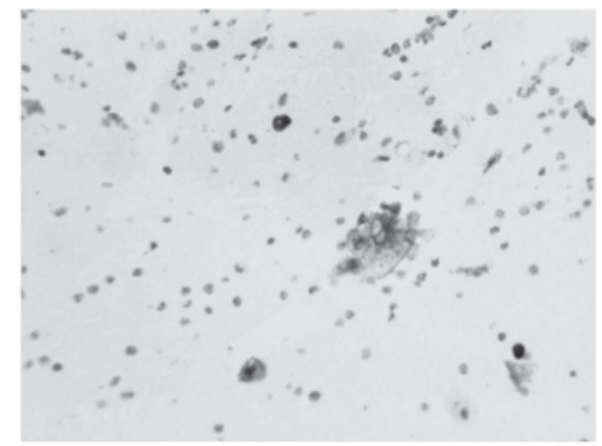

Figure 3. Pathological examination of the thyroid gland resected during surgery. Hematoxylin and eosin staining (magnification, $\mathrm{x} 400$ ).

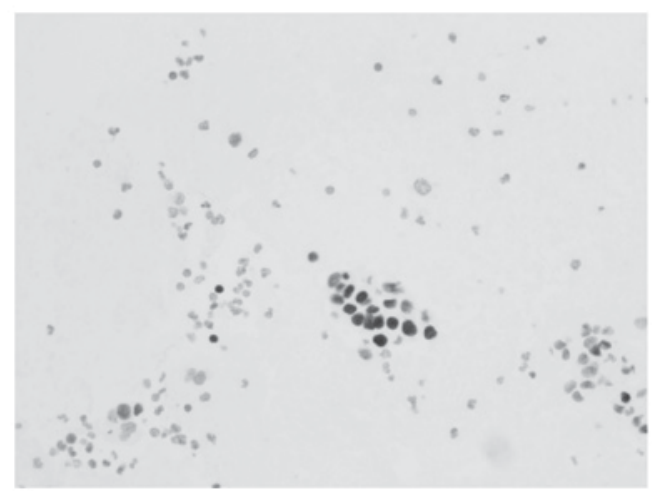

Figure 4. Pathological examination of the thyroid gland resected during surgery. PAX-8 staining (magnification, x200). 
Table I. Antibodies utilized in immunohistochemical staining for renal cell carcinoma.

\begin{tabular}{|c|c|c|c|c|}
\hline Immunohistochemistry antibody & Clone & Dilution & Manufacturer & Cat. no. \\
\hline AE1/AE3 & AE1 \& AE3 & $1: 450$ & Dako $^{a}$ & M3515 \\
\hline CD10 & EP195 & $1: 10$ & Epitomics $^{\mathrm{b}}$ & AC-0169 \\
\hline PAX8 & MRQ-50 & $1: 400$ & Cell Marque ${ }^{c}$ & $363 \mathrm{M}-16$ \\
\hline PN15 & $\mathrm{PN}-15$ & $1: 300$ & Cell Marque ${ }^{c}$ & $329 \mathrm{M}-97$ \\
\hline Vimentin & V9 & $1: 125$ & Dako $^{a}$ & M072529 \\
\hline Cytokeratin 20 & Ks20.8 & $1: 300$ & Dako $^{\mathrm{a}}$ & M701901 \\
\hline Cytokeratin 7 & OV-TL $12 / 30$ & $1: 500$ & Dako $^{a}$ & M701801 \\
\hline Thyroglobulin & Polyclonal & $1: 100,000$ & Dako $^{\mathrm{a}}$ & A025102 \\
\hline Thyroid transcription factor & $8 \mathrm{G} 7 \mathrm{G} 3 / 1$ & $1: 400$ & Dako $^{\mathrm{a}}$ & M357501 \\
\hline Glial fibrillary acidic protein & Polyclonal & $1: 12,000$ & Dako $^{a}$ & Z033401 \\
\hline MNF116 cytokeratin & MNF116 & $1: 150$ & Dako $^{a}$ & M0821 \\
\hline
\end{tabular}

${ }^{\mathrm{a}}$ Agilent technologies Inc., Santa Clara, CA, USA; 'burlingame, CA, USA; ${ }^{\mathrm{C}}$ Rocklin, CA, USA. AE1/3, cytokeratin 1/3; CD, cluster of differentiation; PAX8, paired box gene 8; PN-15, renal cell carcinoma.

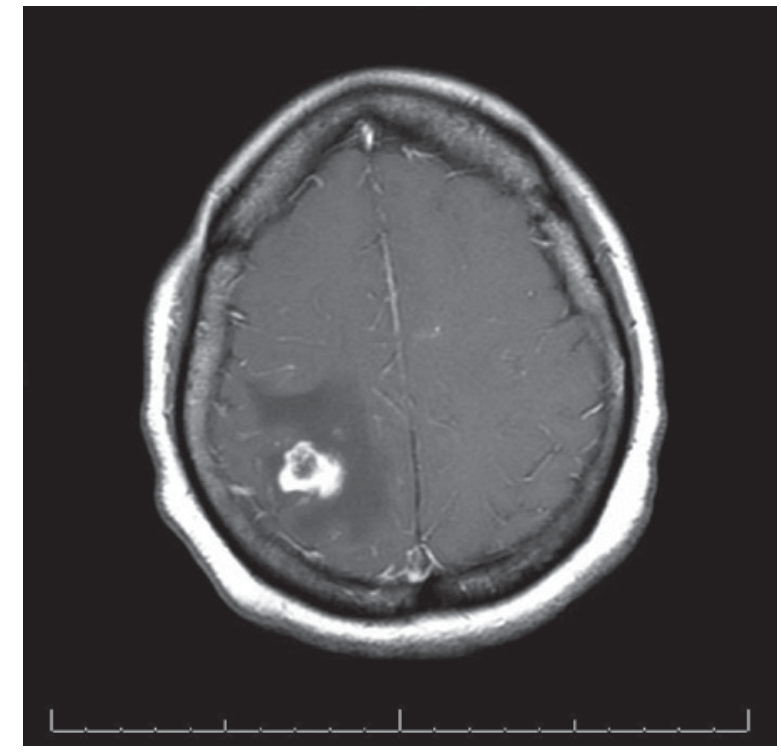

Figure 5. Magnetic resonance imaging of the brain with contrast depicting a right posterior mass with vasogenic edema.

Detection was carried out using DAKO EnVision ${ }^{\mathrm{TM}}$ Flex+ and DAKO Austostainer Link (Dako; Agilent Technologies, Inc.). All antibody details are illustrated in Table I. Metastatic involvement of the thyroid was determined to be associated with the patient's prior history of grade III RCC. In addition to being referred to radiation-oncology and neurology-oncology surgery, the patient was placed on Keppra, remained seizure free and was started on pazopanib as an outpatient.

Several months after discharge, the patient was readmitted to the Miami Valley Hospital for worsening headaches, nausea and vomiting. CT and MRI scans of the brain revealed the recurrence of a $1.9 \mathrm{~cm}$ mass in the right parietal lobe with extensive vasogenic edema (Fig. 5). Biopsy of the mass at several distinct points indicated a vascular lesion composed of numerous vessels of varying caliper, suggestive of AVM. Immunostaining revealed the mass to be AE1/3, cytokeratin

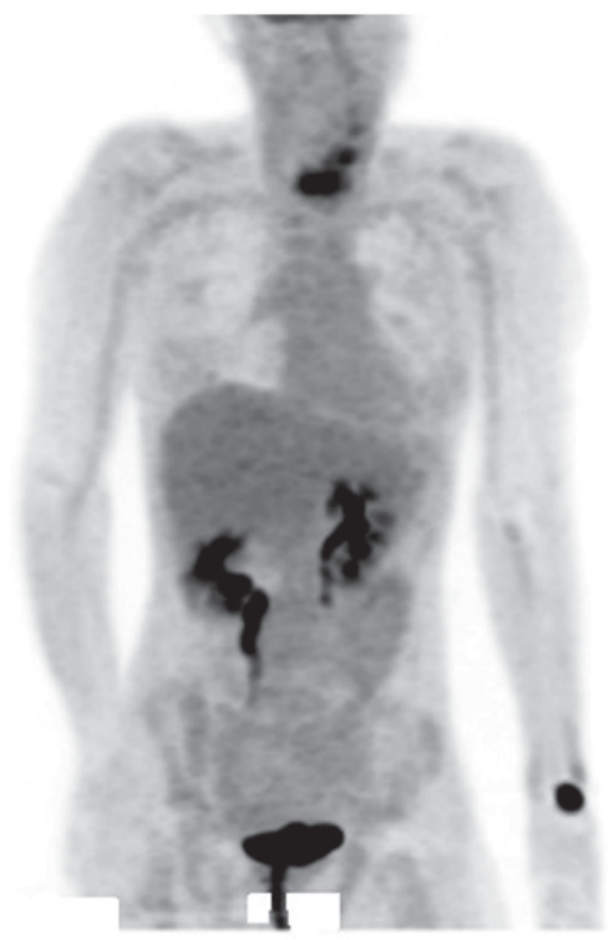

Figure 6. Monochrome positron emission tomography performed during initial evaluation of suspected renal clear cell carcinoma metastasis.

(MNF116; 1:150 dilution; catalog no. M0821; Agilent Technologies), PAX8 and PN-15 negative. The patient was readmitted again to the hospital after several months for worsening headaches, nausea and vomiting. CT and MRI scans of the brain revealed the return of a $3.7 \mathrm{~cm}$ mass exhibiting vasogenic edema. Biopsy of this novel mass did not suggest an AVM, rather the immunohistochemical stains demonstrated the following: Glial fibrillary acidic protein, negative; vimentin, CD10, PN-15, PAX-8 and cytokeratin AE1/3, positive; CK7 and CK20, negative. The findings from the novel lesion supported a diagnosis of metastatic RCC. Fig. 6 presents PET imaging, which suggested metastasis to the thyroid. 


\section{Discussion}

RCC accounts for $<5 \%$ of all malignant tumors (9). Metastasis is common in patients with RCC, with typical sites including the lungs, lymph nodes, bone, and liver (10). Metastasis to the head and neck region is less common (11). To the best of our knowledge, RCC metastasis to the brain associated with AVM formation has not been reported in the literature to date. More commonly, AVMs associated with RCC are located in the renal vasculature and present with the cardinal symptom of flank pain (5). Recent literature has commented on the association between AVMs and RCC as they share highly vascular characteristics (5). It was previously considered that various types of cancer have high circulating expression levels of plasma vascular endothelial growth factor (VEGF) (12). However, a recent study demonstrated that free VEGF plasma levels are low or absent in numerous types of cancer, with the exception of RCC (13). RCC is a vascular tumor that has been demonstrated to lead to the abnormal expression of various angiogenesis-promoting growth factors (14). VEGF is the central pro-angiogenic agent involved in RCC carcinogenesis, and is associated with mutations in the von Hippel-Lindau tumor suppressor (VHL) gene (14). Somatic VHL mutations have been associated with persistently high expression levels of hypoxia-inducible factor-1 $\alpha$ signaling pathway proteins, and with VEGF expression in patients with metastatic RCC (15).

Recent studies using animal models have postulated that genetic manipulation and angiogenic stimulation are each required for AVM development, and are independent of congenital etiologies (8). It may be theorized that the dysregulation of pro-angiogenic factors associated with RCC development contributes to the development of AVMs observed in this type of malignancy. These angiogenic factors are vital to the pathophysiological pathway that leads to RCC, and may well explain the development of AVMs within these neoplasms as demonstrated in the aforementioned case. DNA sequencing of AVM tissue from a patient with Proteus-like syndrome has revealed PTEN mutations that have been previously implicated in Cowden's syndrome (16). With the use of immunohistochemical staining on tissue samples from similar areas of the brain, the progression of negative markers for malignancy in the setting of an AVM to positive markers for malignancy in the absence of AVM was demonstrated. It has been noted that certain pathologists have stated that an AVM and RCC may be regarded as associated entities, rather than distinct processes, in the context of surgical histopathology. Conversely, the case has been presented for serial MR imaging of supposed AVMs in order to distinguish them from malignancies, specifically RCC (5).

The patient in the current case was further unusual in that they developed biopsy-confirmed renal metastasis to the thyroid. Typically, patients with RCC metastasis to the thyroid are detected following a physical exam, not in the setting of a coinciding metastasis to the brain (17). Metastases to the thyroid have generally been revealed to occur several years following nephrectomy (10). It is unclear why metastasis to the thyroid is a rare event, as it has a rich vascular supply (18).

Current guidelines pertaining to metastatic RCC do not recommend routine brain imaging for surveillance purposes unless there are concurrent CNS symptoms or other abnormalities indicating brain involvement (19). Patients with a history of tobacco abuse and pulmonary metastases are more likely to have metastasis to the brain; therefore, this may serve as an indication for surveillance imaging of the brain (19). With increasing understanding of the role of angiogenic dysregulation in the pathogenesis of RCC, novel therapeutic strategies are utilizing targeted anti-angiogenic agents with varying degrees of success (20).

In conclusion, brain metastasis must be considered in patients with a positive history of RCC, who present with an acute onset neurological event and apparent AVM on initial biopsy. Postoperative distinction between AVM and metastasis to the brain may be difficult to distinguish, as discussed in the aforementioned case. A high index of suspicion for brain metastasis, despite initial negative immunohistochemical testing, should be followed up by a repeat biopsy of the tissue in question at a later date. This may assist in guiding palliative therapy should the otherwise prognostically favorable AVM be determined to be a prognostically unfavorable metastasis to the brain.

\section{References}

1. Thoenes W, Störkel S, Rumpelt HJ and Moll R: Cytomorphohogical typing of renal cell carcinoma-a new approach. Eur Urol 18 (Suppl 2): S6-S9, 1990.

2. Nakhjavani MK, Gharib H, Goellner JR and van Heerden JA: Metastasis to the thyroid gland. A report of 43 cases. Cancer 79: 574-578, 1997.

3. National Cancer Institute: Cancer statistics: SEER Stat Fact Sheets: Kidney and renal pelvis cancer. https://seer.cancer. gov/statfacts/html/kidrp.html. Accessed May 1, 2016.

4. Wong C, Leveillee RJ, Yrizarry JM and Kirby K: Arteriovenous malformation mimicking a renal-cell carcinoma. J Endourol 16: 685-686, 2002.

5. Volin S, Steinberg P and Mittleider D: Renal cell carcinoma initially presenting as an arteriovenous malformation: A case presentation and a review of the literature. Case Rep Urol 2013: 356819, 2013.

6. Park SB, Cho KS, Lee JH, Jeong YK, Choi SH, Kang BS and Kim JK: Unusual manifestations of renal cell carcinoma. Acta Radiol 49: 839-847, 2008.

7. Young AM, Teo M, Martin SC, Phang I, Bhattacharya JJ and St George EJ: The diagnosis and management of brain arteriovenous malformations in a single regional center. World Neurosurg 84: 1621-1688, 2015.

8. Chen W, Choi EJ, McDougall CM and Su H: Brain arteriovenous malformation modeling, pathogenesis, and novel therapeutic targets. Transl Stroke Res 5: 316-329, 2014.

9. Ohba K, Miyata Y, Mitsunari K, Matsuo T, Mochizuki Y and Sakai H: Left atrial metastasis of renal cell carcinoma: A case report and review of the literature. BMC Res Notes 7: 520, 2014.

10. Bianchi M, Sun M, Jeldres C, Shariat SF, Trinh QD, Briganti A, Tian Z, Schmitges J, Graefen M, Perrotte P, et al: Distribution of metastatic sites in renal cell carcinoma: A population-based analysis. Ann Oncol 23: 973-980, 2012.

11. Rizzo M, Rossi RT, Bonaffini O, Scisca C, Sindoni A, Altavilla G and Benvenga S: Thyroid metastasis of clear cell renal carcinoma: Report of a case. Diagn Cytopathol 37: 759-762, 2009.

12. Longo R and Gasparini G: Challenges for patient selection with VEGF inhibitors. Cancer Chemother Pharmacol 60: 151-170, 2007.

13. Niers TM, Richel DJ, Meijers JC and Schlingemann RO: Vascular endothelial growth factor in the circulation in cancer patients may not be a relevant biomarker. PLoS One 26: e19873, 2001.

14. Clifford SC, Prowse AH, Affara NA, Buys CH and Maher ER: Inactivation of the von Hippel-Lindau (VHL) tumour suppressor gene and allelic losses at chromosome arm 3p in primary renal cell carcinoma: Evidence for a VHL-independent pathway in clear cell renal tumourigenesis. Genes Chromosomes Cancer 22: 200-209, 1998.

15. Falcão MS, Vinagre J, Soares P, Lopes JM, Brandão E and Carneiro AM: A clear cell renal cell carcinoma inhibiting the response to intravitreal antivascular endothelial growth factor therapy in wet age-related macular disease. Case Rep Ophthalmol 3: 443-451, 2012. 
16. Zhou XP, Marsh DJ, Hampel H, Mulliken JB, Gimm O and Eng C: Germline and germline mosaic PTEN mutations associated with a Proteus-like syndrome of hemihypertrophy, lower limb asymmetry, arteriovenous malformations and lipomatosis. Hum Mol Genet 9: 765-768, 2000.

17. Lee MW, Batoroev YK, Odashiro AN and Nguyen GK: Solitary metastatic cancer to the thyroid: A report of five cases with fine-needle aspiration cytology. Cytojournal 4: 5, 2007.

18. Medas F, Calò PG, Lai ML, Tuveri M, Pisano G and Nicolosi A: Renal cell carcinoma metastasis to thyroid tumor: A case report and review of the literature. J Med Case Rep 7: 265, 2013.
19. Hanzly M, Abbotoy D, Creighton T, Diorio G, Mehedint D, Murekeyisoni C, Attwood K, Kauffman E, Fabiano AJ and Schwaab T: Early identification of asymptomatic brain metastases from renal cell carcinoma. Clin Exp Metastasis 32: 783-788, 2015.

20. Bastos DA, Molina AM, Hatzoglou V, Jia X, Velasco S, Patil S, Voss MH, Feldman DR and Motzer RJ: Safety and efficacy of targeted therapy for renal cell carcinoma with brain metastasis. Clin Genitourin Cancer 13: 59-66, 2015. 